\title{
Tensiones residuales en uniones soldadas por FSW en aluminio 7075-T651
}

\author{
(Residual stresses in 7075-T651 FSW joint)
}

\author{
Leonardo N. Tufaro ${ }^{1,2}$, Alejandro Burgueño ${ }^{1}$, Hernán G. Svoboda ${ }^{2,3}$ \\ ${ }^{1}$ Instituto Nacional de Tecnología Industrial, Centro de Investigación y Desarrollo en Mecánica, San Martín, Buenos Aires, \\ Argentina,ltufaro@inti.gob.ar \\ ${ }^{2}$ Universidad de Buenos Aires, Laboratorio de Materiales y Estructuras, INTECIN, Facultad de Ingeniería, Ciudad de Buenos Aires, \\ Argentina,hsvobod@fi.uba.ar \\ ${ }^{3}$ Universidad Consejo Nacional de Investigaciones Cientificas y Técnicas, Ciudad de Buenos Aires, Argentina
}

\begin{abstract}
Resumen
Las tensiones residuales pueden ser un aspecto de relevancia en la integridad estructural de componentes en servicio, pudiendo presentar una importante influencia sobre la vida a la fatiga, entre otros mecanismos de falla. El proceso de soldadura por fricciónagitación (FSW) ha revolucionado en los últimos años el campo de la tecnología de la soldadura. Su mayor aplicación se ha dado en las aleaciones de aluminio, aunque hoy en día se utiliza para prácticamente todos los materiales. Una de las ventajas enunciadas de este proceso es el menor nivel de tensiones residuales resultantes, debido a que se produce en estado sólido por lo que los gradientes térmicos durante la soldadura son menores. Entre las aleaciones de aluminio de uso estructural, las aleaciones termoenvejecibles de la serie $7 X X X$ se utilizan habitualmente en la industria aeronáutica y aeroespacial debido a su alta resistencia mecánica. El objetivo del presente trabajo es analizar el efecto de la velocidad de avance en FSW sobre las tensiones residuales en juntas de aluminio AA7075-T651, mediante la técnica de seccionamiento. Se soldaron probetas de dicha aleación de 150x150x4mm mediante FSW, variando la velocidad de avance entre 51 y $206 \mathrm{~mm}^{\mathrm{min}}{ }^{-1}$. Durante la soldadura se adquirieron los ciclos térmicos. Posteriormente se midieron las tensiones residuales longitudinales a distintas distancias del cordón de soldadura, en cada caso. A partir de los ciclos térmicos adquiridos se obtuvieron los gradientes térmicos en la zona de medición. Las tensiones residuales máximas se encontraron entre 52 y $78 \mathrm{MPa}$, correspondiente entre 10 y $15 \%$ del límite de fluencia del material. Las mismas aumentaron con la velocidad de avance, consistentemente con un aumento en el gradiente térmico observado. Dichos valores de tensiones residuales son menores que los obtenidos para la soldadura de estos materiales mediante procesos del tipo GMAW.
\end{abstract}

Palabras-clave: FSW; tensiones residuales; aluminio AA 7075-T651.

\begin{abstract}
Residual stresses could be a relevant issue in the structural integrity of a component in service and usually have a major influence on their fatigue life, among other failure mechanisms. In recent years Friction-Stir Welding (FSW) has revolutionized the welding technology. Its main application has been given in aluminum alloys, but nowadays can be applicable to different materials. One of the several advantages of this process is a lower level of residual stresses, because the thermal gradients introduced during welding are lower. Among aluminum alloys used for structural applications, $7 X X X$ strain-aged series are usually employed in the aerospace industry, because of their high strength. The aim of this paper is to analyze the effect of welding speed of FSW on residual stresses in aluminum AA7075-T651 joints, using sectioning technique. Coupons were welded AA7075-T651 FSW of 150x150x4 mm by varying the welding speed between 51 and $206 \mathrm{~mm} . \mathrm{min}^{-1}$. During the welding thermal cycles were acquired. Subsequently, longitudinal residual stresses measured at different distances from the weld, in each case. Based on the acquired thermal cycling thermal gradients were obtained in the measurement area. Maximum residual stresses ranged from 52 to $78 \mathrm{MPa}$, corresponding to a range of 10 and $15 \%$ of the yield strength. They increased with increasing welding speed, consistent with an increase in the thermal gradient observed. These residual stresses values are lower than those obtained for the welding of these materials by type GMAW processes.
\end{abstract}

Key-words: FSW; residual stress; AA7075-T651 aluminum alloy.

\section{Introducción}

La formación de tensiones residuales en uniones soldadas se

(Recebido em 29/02/2012; Texto final em 07/03/2012).

Artigo originalmente publicado no CONSOLDA 2011 debe a la presencia de campos no-uniformes de deformaciones no-elásticas, como las deformaciones plásticas y térmicas, mientras se efectúa la soldadura. Debido a que la soldadura 
por fricción-agitación (FSW) es un proceso en estado sólido, las temperaturas alcanzadas son menores a la temperatura de fusión. Por este motivo, generalmente se espera que las tensiones residuales sean menores a las generadas en soldaduras por fusión. Sin embargo, es necesaria una sujeción más rígida de las piezas a unir, que restringe la contracción durante el enfriamiento, generando un aumento en las tensiones residuales por esta causa [1].

Se ha encontrado que las tensiones residuales presentes en uniones soldadas por FSW suelen presentar una distribución en forma de "M", siendo las tensiones longitudinales, levemente superiores que las transversales. Si bien esta distribución es prácticamente simétrica, las tensiones suelen ser levemente mayores del lado de avance (AS) [2,3].

En cuanto a la influencia de las variables de soldadura, se ha reportado que las tensiones longitudinales aumentan con la velocidad de avance [1,4] y al disminuir la velocidad de rotación [4], aunque otros autores han reportado que no se ven afectadas sensiblemente por este parámetro [2]. En este sentido, la magnitud de las tensiones residuales medidas, si bien se encuentran en el mismo orden, presentan variaciones de relativa importancia en función de otros aspectos como la geometría de la herramienta. Se ha intentado correlacionar la tensión residual con el calor aportado neto [2,5], reportándose una tendencia a disminuir el valor de dicha tensión residual con el aumento del aporte térmico, si bien la correlación es relativamente baja.

En estos últimos años se han realizado distintos trabajos sobre uniones soldadas por FSW de AA 7075-T651. Las propiedades mecánicas de la serie 7XXX varían con el tiempo, debido a que endurecen por precipitación a temperatura ambiente (envejecimiento natural), luego de producirse la soldadura por FSW [3], encontrándose variaciones temporales de la dureza, la resistencia y las tensiones residuales. Dichas tensiones residuales en la zona agitada (WN) disminuyen, mientras que las presentes en la zona afectada por el calor (HAZ) aumentan con el tiempo [3]. Por otra parte, la resistencia a la tracción y la dureza aumentan con el tiempo [6,7].

Las tensiones residuales resultantes de la soldadura se encuentran vinculadas a los parámetros del proceso utilizados a través del campo térmico que este conjunto de parámetros genera sobre la probeta. En este sentido, son escasos los estudios experimentales que vinculen estos aspectos de modo de lograr una mejor comprensión de los fenómenos actuantes, en particular sobre aleaciones termoenvejecibles de uso estructural. El objetivo del presente trabajo es analizar el efecto de la velocidad de avance en soldadura FSW sobre las tensiones residuales longitudinales en uniones de aluminio AA7075-T651, analizando los ciclos térmicos generados.

\section{Materiales y Métodos}

\subsection{Material}

En el presente trabajo se estudió una aleación de aluminio AA7075-T651 en chapa de $4 \mathrm{~mm}$ de espesor. Se realizó un ensayo de tracción según la norma ASTM E8M-04 [8] a fin de determinar las propiedades mecánicas del material. El límite convencional fue de $\sigma_{0,2}=526 \mathrm{MPa}$, la resistencia a la tracción $\sigma_{\text {UTS }}=576 \mathrm{MPa}$, un alargamiento a rotura de $\mathrm{A}=12 \%$, para una longitud de referencia de $50 \mathrm{~mm}$. El módulo de elasticidad determinado fue de E=71 GPa. Este último y el coeficiente de Poisson, $\mathrm{n}=0,33$ [9], se utilizaron para el cálculo de las tensiones residuales a partir de las deformaciones medidas. Por último, para determinar la potencia y calor aportado neto que surgen del ajuste de los ciclos térmicos adquiridos se considera una densidad de $2800 \mathrm{~kg} . \mathrm{m}^{-3}$, una conductividad térmica de 130 $\mathrm{W} \cdot \mathrm{m}^{-1}$ y una calor específico de $960 \mathrm{~J} \cdot \mathrm{kg}^{-1} \cdot \mathrm{K}^{-1}[9]$.

\subsection{Ejecución de Soldadura y Adquisición de Ciclos Térmicos}

Se soldaron probetas de AA7075-T651 de 150x75x4 mm a tope con el proceso FSW utilizando una máquina herramienta adaptada. En la Tabla 1 se muestran los parámetros empleados para las cinco probetas soldadas. La velocidad de avance se varió desde $51 \mathrm{~mm} . \mathrm{min}-1$ hasta $206 \mathrm{~mm} . \mathrm{min}-1$ para una misma velocidad de rotación y ángulo de inclinación.

La herramienta utilizada presenta un pin de geometría cónica, de hombro cóncavo y fue construida en acero para herramientas del tipo H13. El diámetro del hombro es de 12 $\mathrm{mm}$ y los diámetros mayor y menor del pin son de 4 y $3 \mathrm{~mm}$, respectivamente. Por último, la altura del pin es de $3,8 \mathrm{~mm}$.

Tabla 1. Parámetros de soldadura por FSW

\begin{tabular}{|c|c|c|c|c|}
\hline Designación & $\begin{array}{c}\text { Velocidad de } \\
\text { avance } \\
\mathrm{U}\left(\mathrm{mm}^{\mathrm{m}} \mathrm{min}^{-1}\right)\end{array}$ & $\begin{array}{c}\text { Velocidad } \\
\text { de } \\
\text { rotación } \\
\omega(\mathrm{rpm})\end{array}$ & $\begin{array}{c}\text { Ángulo de } \\
\text { inclinación } \\
\left({ }^{\circ}\right)\end{array}$ & $\begin{array}{c}\text { Pitch }=\mathrm{w} / \mathrm{U} \\
\left(\mathrm{rev} \cdot \mathrm{mm}^{-1}\right)\end{array}$ \\
\hline 51 & 51 & & & 10,1 \\
\hline 73 & 73 & \multirow{2}{*}{514} & \multirow{2}{*}{2,0} & 7,0 \\
\hline 98 & 98 & & & 5,2 \\
\hline 146 & 146 & & & 3,5 \\
\hline 206 & 206 & & & 2,5 \\
\hline
\end{tabular}

La medición de ciclos térmicos durante la soldadura se realizó con tres termocuplas tipo K, las que se ubicaron en la mitad de la longitud de la probeta, en el lado de retroceso. La primera se posicionó a aproximadamente $1 \mathrm{~mm}$ del borde del cordón de soldadura y las otras dos en una posición correspondiente a la posición de los extensómetros. En la Figura 1.A se observa la posición de las termocuplas (TC) y el sistema de soldadura por FSW. En la Figura 1.B se observa una probeta soldada por FSW.

\subsection{Medición de Tensiones Residuales}

Para la evaluación de las tensiones residuales se utilizó el método de seccionamiento [10,11]. Se instrumentaron 4 extensómetros eléctricos (SG) lineales con una resistencia de $119,4 \mathrm{~W}$ y un factor de gage de 2,43, en cada probeta. En mediciones preliminares se verificó que con dos cortes 


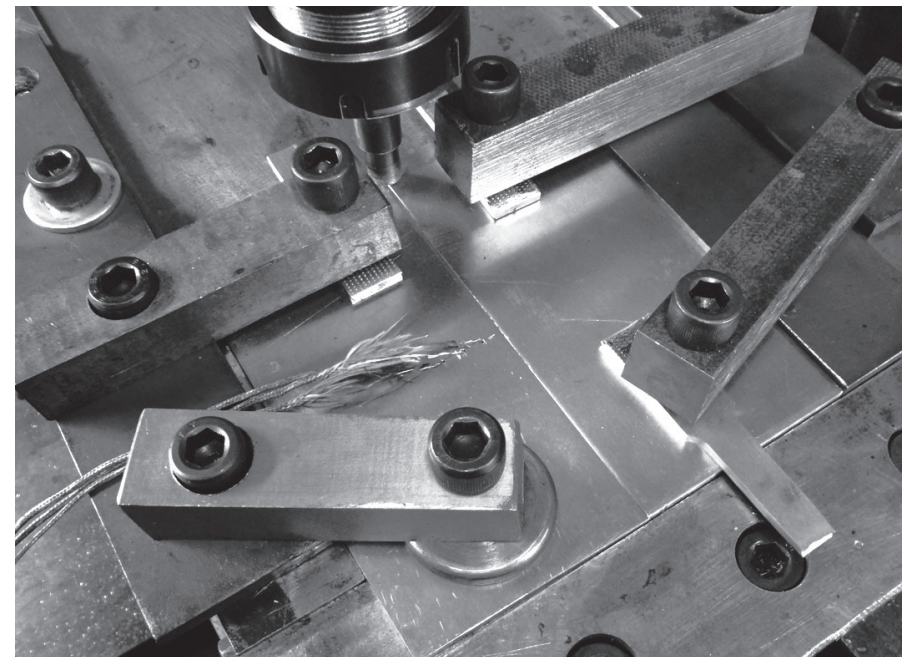

(a)

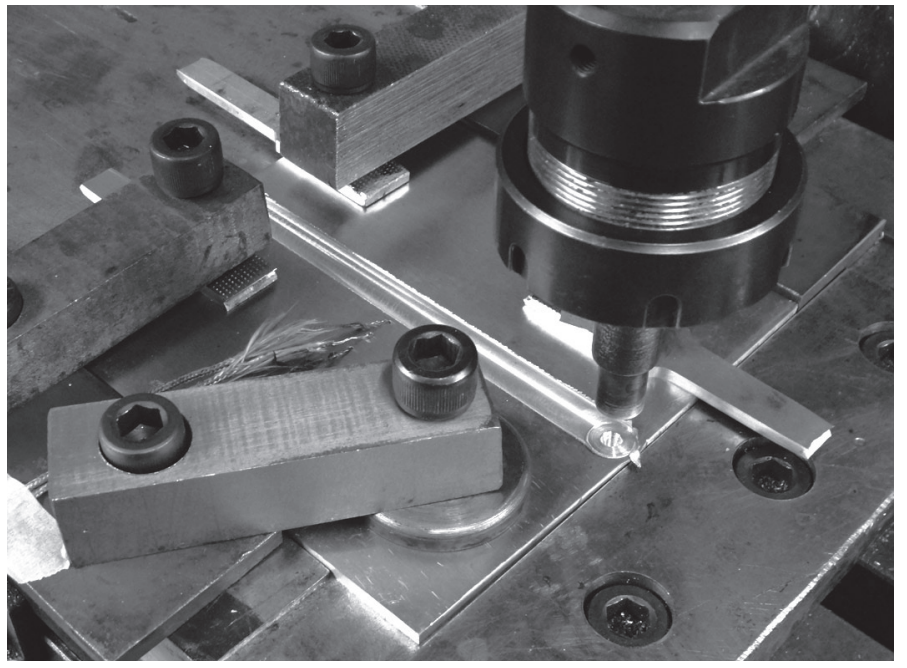

(b)

Figura 1. A. Ubicación de las TC para la adquisición de ciclos térmicos, B. Soldadura por fricción-agitación (FSW)

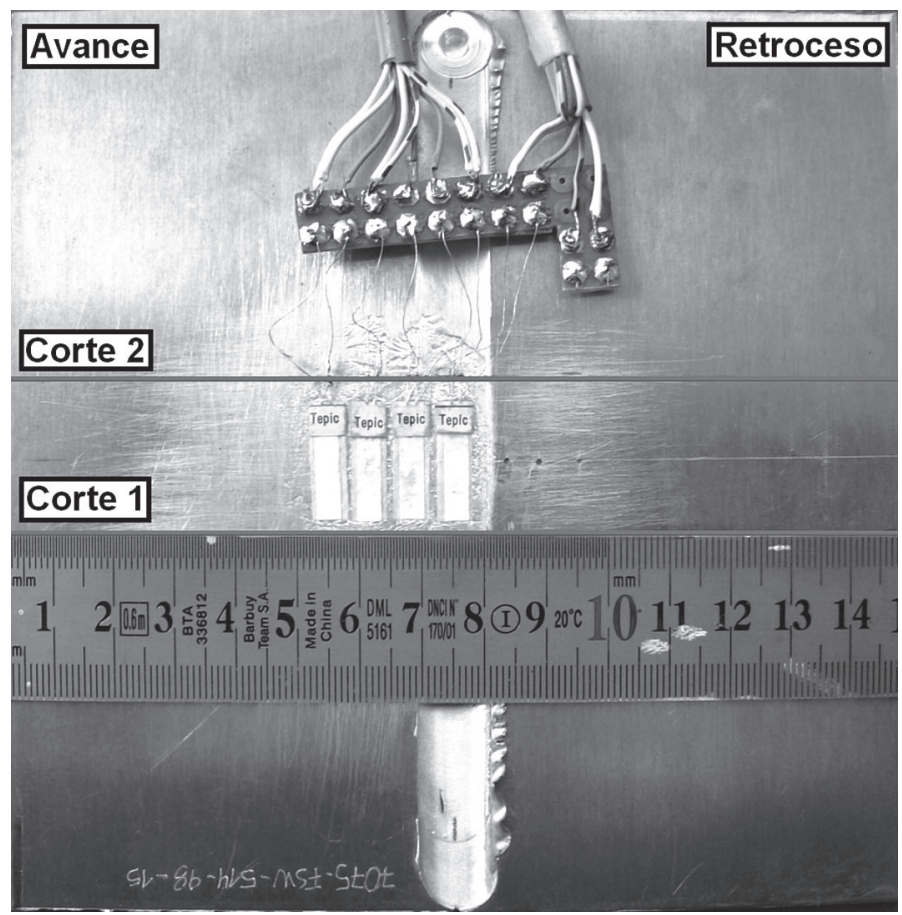

(a)

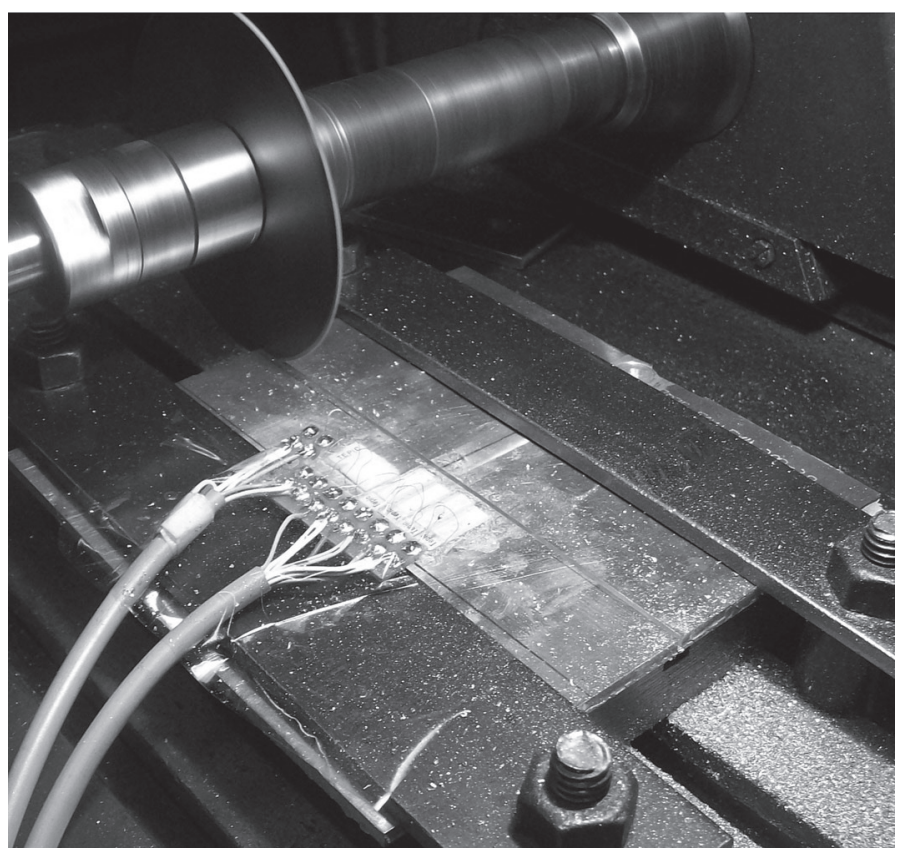

(b)

Figura 2. A. Localización de SG y secuencia de corte, B. Seccionamiento de probetas con fresadora horizontal

puede considerarse que las tensiones se relajan en su totalidad, validándose la técnica experimental empleada. Los extensómetros se ubicaron a la mitad de la longitud de la probeta, en dirección paralela al cordón de soldadura del lado de avance. El primero se posicionó sobre el centro del cordón y los otros tres uno a continuación del otro como lo muestra la Figura 2.A, a fin de obtener información sobre la variación de las tensiones residuales con la distancia al cordón.

Para la instalación de los extensómetros se preparó la superficie de cada probeta. Se pegaron los extensómetros e instrumentaron siguiendo las recomendaciones del fabricante [12].

Los cortes se realizaron es una fresadora horizontal con una fresa de disco de $1 \mathrm{~mm}$ de espesor. La implementación de esta técnica de corte se puede observar en la Figura 2.B. Hay que resaltar que todos los procesos con arranque de viruta introducen tensiones residuales en la superficie. Sin embargo, si el tamaño del elemento en el que se miden las deformaciones es más grande que aproximadamente 2 á $3 \mathrm{~mm}$, a continuación no es necesario aplicar alguna técnica de remoción de material para eliminar dichas tensiones residuales superficiales [13].

Para la medición de las deformaciones asociadas al relevamiento de las tensiones residuales se empleó un equipo Vishay P-3500.

Considerando como afecta el tiempo de envejecimiento a las propiedades mecánicas de la unión soldada es que todas las 


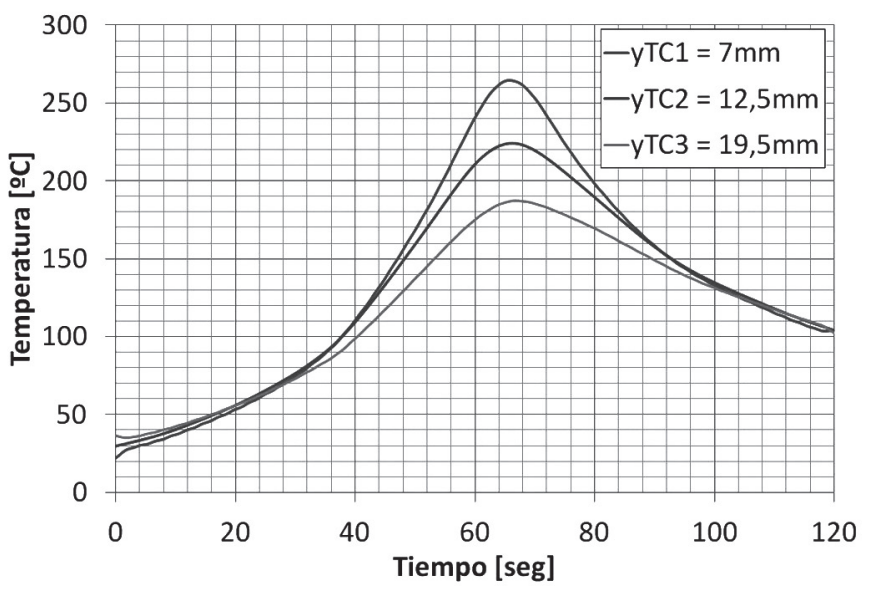

(a)

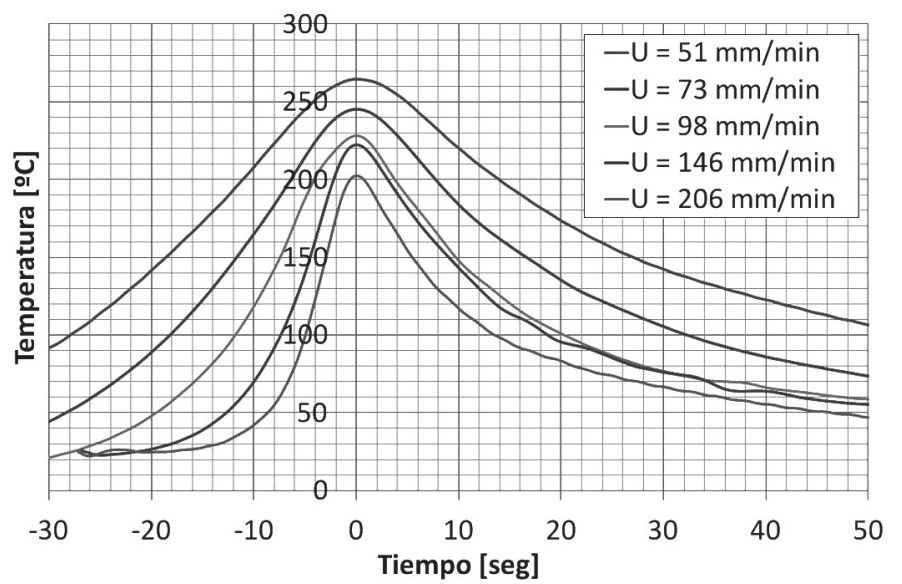

(b)

Figura 3. A. Ciclos térmicos de las tres TC de la probeta 51, B. Comparación de ciclos térmicos para las probetas soldadas con las distintas velocidades de avance, medidos a $1 \mathrm{~mm}$ del borde

mediciones de las tensiones residuales se realizaron luego de entre 31 y 36 días posteriormente a la soldadura, con la intención eliminar esa variable.

\subsection{Macrografías}

De cada probeta seccionada se extrajo un corte transversal el cual se preparó para observación macrográfica. Se observaron las distintas zonas de las uniones soldadas, así como la presencia de discontinuidades asociados al procedimiento.

\section{Resultados y Discusión}

Como pudo verse en la Figura 1.B se obtuvieron juntas soldadas de muy buen aspecto superficial, con buena penetración y sin defectos superficiales apreciables, para todas las condiciones de velocidad de avance analizadas.

\subsection{Ciclos Térmicos}

La Figura 3.A muestran los ciclos térmicos adquiridos por las distintas termocuplas, con las distancias al centro del cordón, para la soldadura de la probeta 51. Se puede apreciar como las temperaturas disminuyen con la distancia al borde del cordón.

En la Figura 3.B se grafican los ciclos térmicos de las distintas probetas soldadas, correspondientes a la termocupla más cercana al borde del cordón $(1 \mathrm{~mm})$. Se observa que al aumentar la velocidad de avance las temperaturas disminuyen, y que las velocidades de calentamiento y de enfriamiento aumentan.

La potencia neta $\mathrm{Q}$ y el calor aportado neto $\mathrm{H}$ se determinan a partir de la medición de temperaturas. Por lo tanto, estos son parámetros netos y no es necesario hacer ninguna consideración sobre el rendimiento del proceso. Para obtener la potencia neta $\mathrm{Q}$ se ajustaron los ciclos térmicos medidos con la expresión de Rosenthal de transmisión de calor bidimensional correspondiente a una fuente de calor puntual que avanza a una velocidad constante sobre una placa infinita, representada en la Ecuación 1 [14].

$$
T-T_{0}=\frac{Q}{2 \pi \cdot k \cdot b} \cdot e^{\frac{-U \cdot x}{2 \alpha}} \cdot Y_{0}\left(\sqrt{x^{2}+y^{2}} \cdot \sqrt{\frac{U^{2}}{4 \alpha^{2}}+\frac{2 h_{c}}{k \cdot b}}\right)
$$

En la Tabla 2 se presentan los resultados obtenidos y calculados a partir de los ciclos térmicos adquiridos, para las cinco juntas soldadas: temperatura máxima, potencia neta y calor aportado neto.

Tabla 2. Resultados obtenidos para las distintas probetas soldadas: Temperatura máxima, Potencia neta, Calor aportado neto

\begin{tabular}{|c|c|c|c|}
\hline Designación & $\begin{array}{c}\text { Temperatura } \\
\text { máxima } \\
\mathrm{T}_{\text {max }}\left({ }^{\circ} \mathrm{C}\right)\end{array}$ & $\begin{array}{c}\text { Potencia } \\
\text { neta } \\
\mathrm{Q}(\mathrm{W})\end{array}$ & $\begin{array}{c}\text { Calor aportado } \\
\text { neto } \\
\mathrm{H}=\mathrm{Q} / \mathrm{U}(\mathrm{J} / \mathrm{mm})\end{array}$ \\
\hline 51 & 265 & 625 & 735 \\
\hline 73 & 245 & 633 & 520 \\
\hline 98 & 228 & 617 & 378 \\
\hline 146 & 223 & 657 & 270 \\
\hline 206 & 203 & 613 & 179 \\
\hline
\end{tabular}

Se puede observar que las temperaturas máximas son menores a las reportadas en la literatura [1]. Esto puede deberse a que las dimensiones de la herramienta empleada son menores que las utilizadas habitualmente para este tipo de junta. Un diámetro de herramienta menor implica una potencia neta menor, y por lo tanto, para un mismo espesor de chapa, resultan menores temperaturas. Adicionalmente, en la Figura 4.A se observa que la temperatura máxima y la velocidad de avance presentan una tendencia potencial, obteniéndose un buen grado de ajuste. 


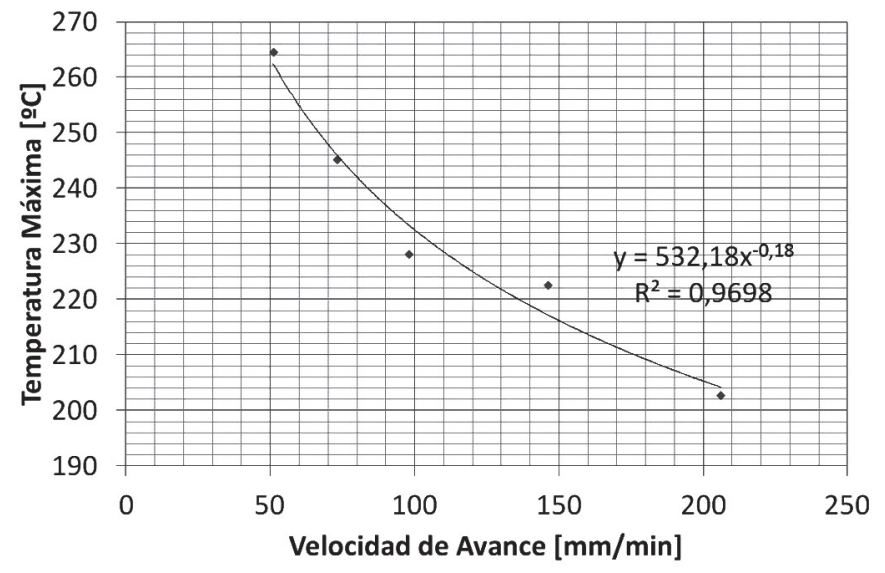

(a)

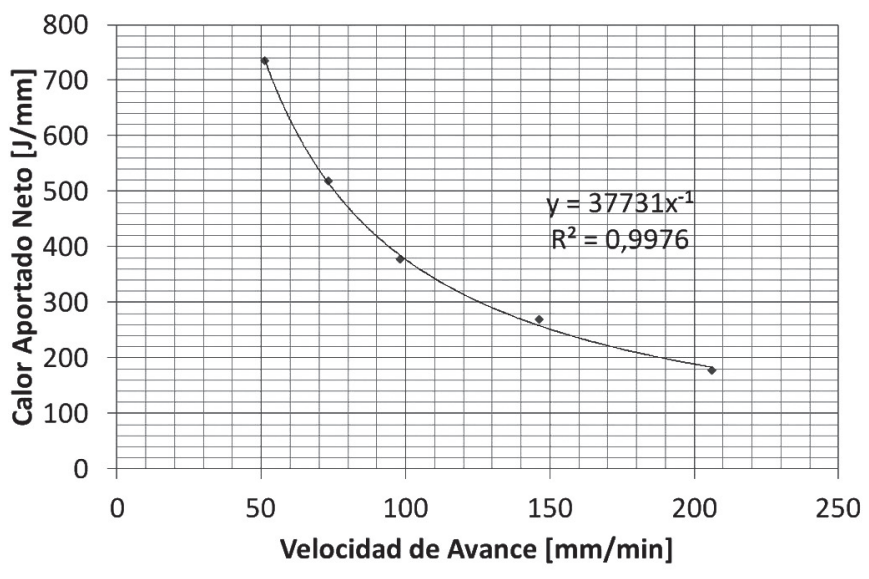

(b)

Figura 4. A. Temperatura máxima en función de la velocidad de avance, B. Calor aportado neto en función de la velocidad de avance

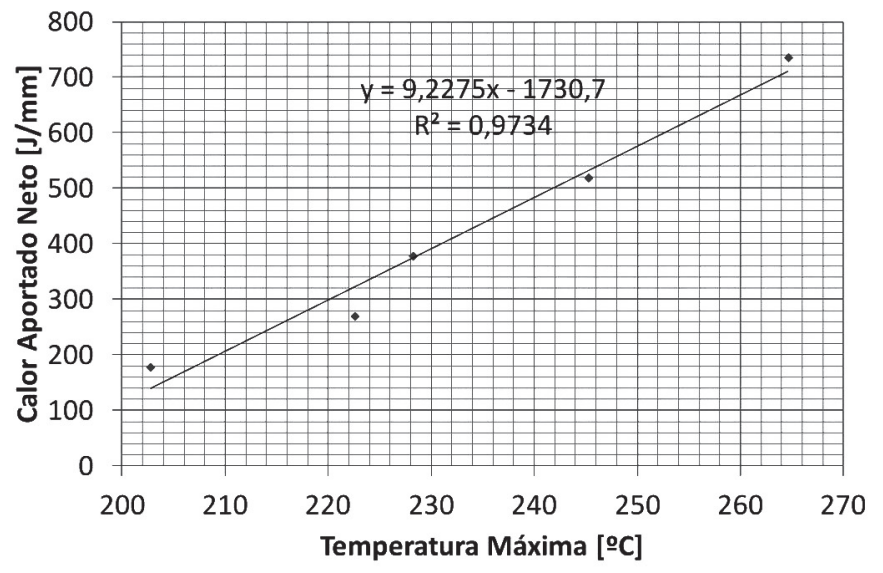

(a)

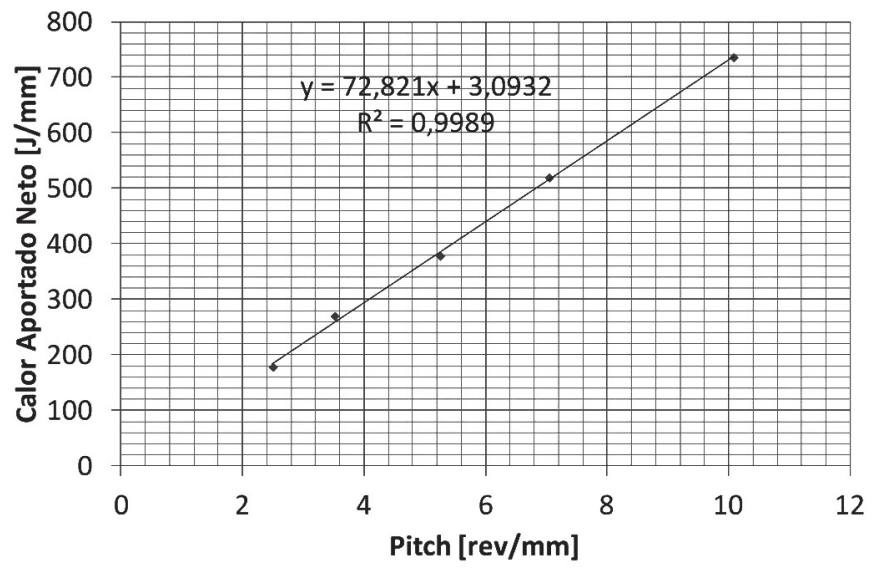

(b)

Figura 5. A. Calor aportado neto en función de la temperatura máxima, B. Calor aportado neto en función del pitch

Se puede apreciar que la potencia neta no varía considerablemente con la velocidad de avance. En consecuencia, la variación del calor aportado neto $\mathrm{H}$, que es la relación entre la potencia neta y la velocidad de avance, está gobernada prácticamente en su totalidad por esta última. Por este motivo, correlacionan muy bien con una tendencia potencial, como se puede observar en la Figura 4.B. Como era de esperarse, el exponente es -1 y el coeficiente 37731 es la potencia neta promedio expresada en J. $\mathrm{min}^{-1}$ que surge del ajuste y resulta ser $629 \mathrm{~W}$.

En la Figura 5.A puede apreciarse que, en concordancia con las dos tendencias de la Figura 4, el calor aportado neto y la temperatura máxima presentan una tendencia lineal, lo cual se ha reportado previamente [15]. Por último, en la Figura 5.B se observa que el calor aportado neto y el pitch correlacionan muy bien con una tendencia lineal. Esto se debe a lo mencionado anteriormente para la Figura 4.B y a que la velocidad de rotación se mantiene constante.

Estas expresiones obtenidas pueden ser de utilidad a fin de lograr una mejor comprensión de la influencia de las distintas variables del proceso y para generar información que permita predecir ciertas características de la junta.
Asimismo, es importante destacar la amplia dispersión de resultados reportados en la literatura, lo cual estaría asociado a variables no consideradas al comparar resultados como la geometría de la herramienta, el ángulo de la herramienta, la fuerza axial, etc.

\subsection{Tensiones Residuales Longitudinales}

A partir de mediciones preliminares para la validación de la técnica de medición se determinó que las deformaciones transversales son despreciables. Por lo tanto, para el cálculo de las tensiones residuales longitudinales se desprecian las deformaciones transversales, y su expresión resulta en la Ecuación 2.

$\sigma=\frac{-E}{1-v^{2}} \cdot \varepsilon$

En la Tabla 3 se muestra la tensión residual longitudinal máxima medida para las distintas probetas soldadas, junto con el tiempo de envejecimiento de cada una. 
Tabla 3. Resultados obtenidos para las distintas probetas soldadas: Tensión residual longitudinal máxima

\begin{tabular}{|c|c|}
\hline Designación & $\begin{array}{c}\text { Tensión residual longitudinal máxima } \\
\sigma_{\text {RLmax }}(\mathrm{MPa})\end{array}$ \\
\hline 51 & 52 \\
\hline 73 & 63 \\
\hline 98 & 73 \\
\hline 146 & 72 \\
\hline 206 & 78 \\
\hline
\end{tabular}

Puede verse un leve incremento de las tensiones residuales medidas con la velocidad de avance, consistentemente con lo reportado en la literatura. A su vez se muestra que el tiempo de envejecimiento natural de las distintas probetas fue prácticamente el mismo, no presentando influencia en ese sentido [1]. Los valores de tensiones residuales medidos se encuentran en el orden de magnitud de los reportados en la literatura para materiales similares $[3,5]$.

En la Figura 6 se grafican los perfiles de tensiones residuales longitudinales medidos, en función de la distancia al centro de la junta. Se puede apreciar que todos los perfiles presentan una distribución y gradientes similares, con la tensión de tracción máxima en el centro del cordón.

El perfil correspondiente a una velocidad de avance de $51 \mathrm{~mm} \cdot \mathrm{min}^{-1}$ es considerablemente menor al resto. Si bien al aumentar la velocidad de avance también lo hacen las tensiones residuales longitudinales, a partir de una velocidad de avance de $73 \mathrm{~mm} . \mathrm{min}^{-1}$ parecen dejar de hacerlo. Sin embargo, las tensiones máximas presentan una mayor tendencia a aumentar con la velocidad de avance que el resto del perfil. Estas tensiones residuales máximas se encuentran entre 52 y $78 \mathrm{MPa}$ correspondiéndose con un 10 a $15 \%$ del límite de fluencia del material.

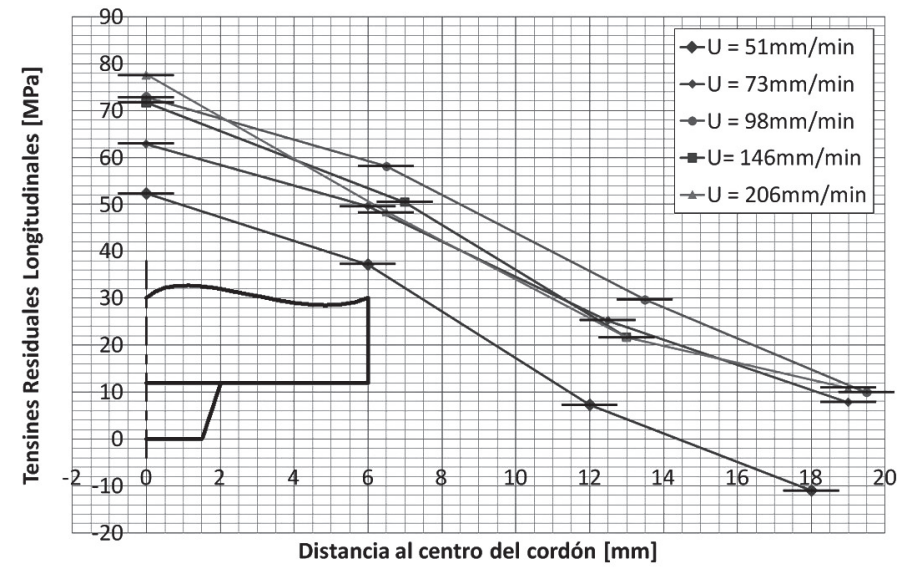

Figura 6. Tensiones residuales longitudinales en función de la distancia al centro del cordón para distintas velocidades de avance.

Este comportamiento levemente diferente observado para las tensiones máximas medidas en el centro del cordón, podría estar asociado a que en esta zona adicionalmente a los mecanismos de formación de tensiones residuales por gradientes térmicos podría existir alguna componente de las tensiones residuales asociada al proceso de deformación plástica severa que tiene lugar en el WN.

Asimismo, el valor de estas tensiones es sensiblemente inferior a lo obtenido para soldadura por fusión $[10,11]$.

La distribución se asemeja a las mediciones realizadas en el trabajo de Linton y Ripley para el aluminio AA 7075-T651 en un tiempo de envejecimiento menor a una semana. Sin embargo, la magnitud de las tensiones residuales obtenidas en aquel trabajo es el doble que las medidas en este trabajo. En ese caso, los parámetros utilizados fueron $350 \mathrm{rpm}$ y $80 \mathrm{~mm} \cdot \mathrm{min}^{-1}$ de velocidades de rotación y avance respectivamente, y un ángulo de inclinación de $0^{\circ}$. Además, la herramienta utilizada era de pin cilíndrico roscado, tenía un diámetro de hombro de $25 \mathrm{~mm}$, y un diámetro de pin de $8 \mathrm{~mm}$, con un espesor de chapa de $6 \mathrm{~mm}$ de espesor [3].

Por otro lado los valores obtenidos en este trabajo son similares, aunque levemente inferiores, a los reportados por Fratini y Zuccarello medidos en AA7075-T6, en donde se observó una tendencia similar con el aporte térmico, utilizando pitch entre 3,75 y 15 [5]. Este trabajo se realizó en chapa de 3 $\mathrm{mm}$ de espesor, con una herramienta de pin cilíndrico, si bien no se indica el diámetro del hombro y el tiempo de envejecimiento natural.

\subsection{Correlaciones entre Tensiones Residuales y Campo Térmico}

La Figura 7.A muestra que la tensión residual longitudinal máxima y el calor aportado neto presentan una tendencia lineal. A partir de la misma se establece que cuanto más fría es la junta mayores son las tensiones residuales. Sin embargo, esta relación debe entenderse con cuidado, ya que en este caso la potencia neta y la velocidad de rotación son invariantes. No debe perderse de vista que, en este caso, un menor calor aportado neto implica una mayor velocidad de avance, y por lo tanto un campo de temperaturas menos uniforme, que resulta en un mayor nivel de tensiones residuales.

En el trabajo de Linton y Ripley se obtuvieron mayores tensiones residuales con un diámetro de hombro de $25 \mathrm{~mm}$, lo que implica una mayor potencia neta para velocidades comparables [3]. En el presente trabajo, al utilizar una herramienta con dimensiones menores a las comúnmente utilizadas, resulta una potencia menor, obteniéndose menores temperaturas máximas y tensiones residuales. Por lo tanto, el diseño de la herramienta es un factor fundamental en la generación de tensiones residuales.

De los coeficientes obtenidos del ajuste de la Figura 7.A se obtiene una expresión para correlacionar la tensión máxima con la velocidad de avance, que se grafica en la Figura 7.B y se muestra en la Ecuación 3.

$\sigma(\mathrm{U})=\left(-0,0446 \frac{\mathrm{mm}}{\mathrm{J}} \cdot \frac{629 \mathrm{~W}}{\mathrm{U}}+86,044\right) \mathrm{MPa}$

Se puede apreciar claramente que la tensión residual longitudinal máxima aumenta con la velocidad de avance. Sin 


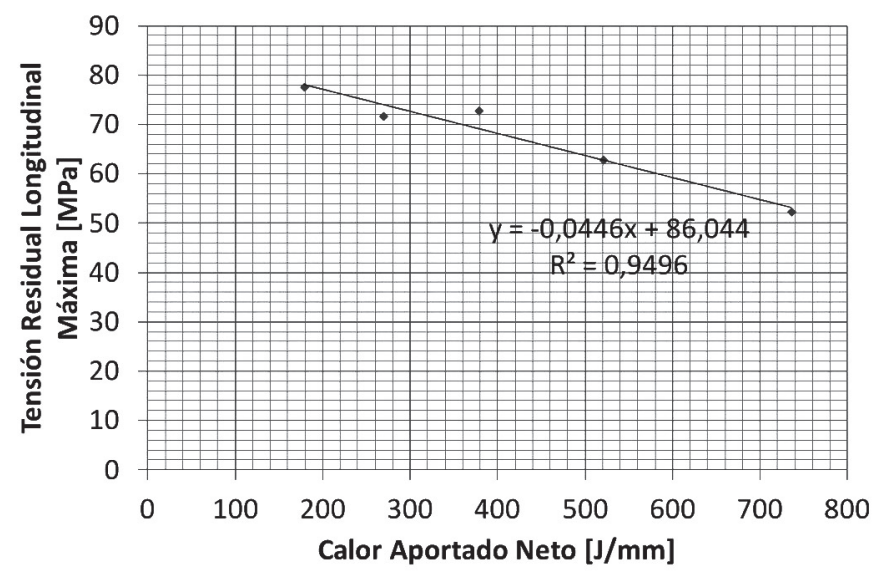

(a)

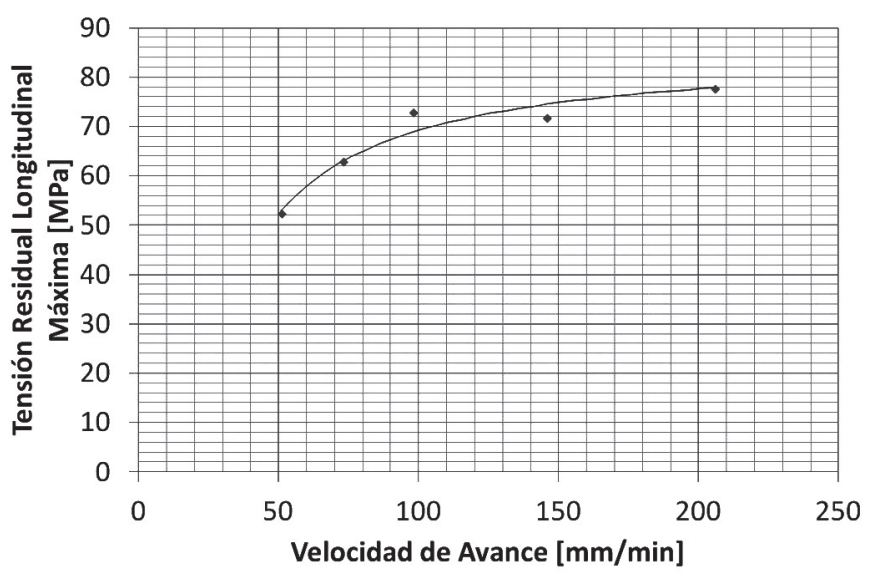

(b)

Figura 7. A. Tensión residual longitudinal máxima en función del calor aportado neto, B. Tensión residual longitudinal máxima en función de la velocidad de avance

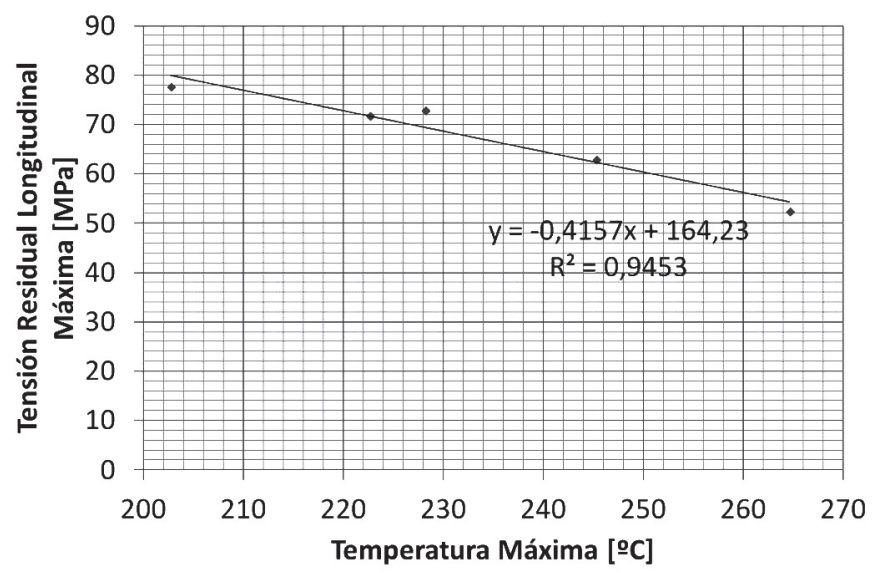

(a)

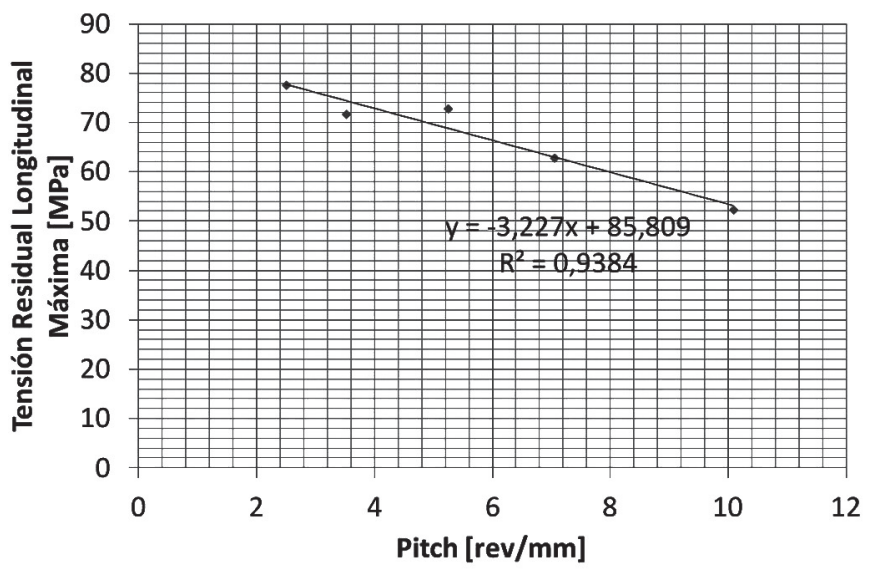

(b)

Figura 8. A. Tensión residual longitudinal máxima en función de la temperatura máxima, B. Tensión residual longitudinal máxima en función del pitch

embargo, a partir de $100 \mathrm{~mm} \cdot \mathrm{min}^{-1}$ la tensión residual máxima presenta un aumento marginal.

En correspondencia con la relación lineal anteriormente apreciada entre el calor aportado neto y la temperatura máxima, una tendencia lineal entre esta última y la tensión máxima se muestra en la Figura 8.A. Si bien esta relación permite una mejor comprensión del fenómeno físico que tiene lugar, dado que el calor aportado neto es un parámetro directamente relacionable con las variables del proceso presenta tal vez una perspectiva de mayor utilidad tecnológica.

En la Figura 8.B puede apreciarse que, en concordancia con la tendencia lineal entre el calor aportado neto y el pitch observada en la Figura 5.B, este último y la tensión residual máxima presentan una correlación lineal y una gráfica muy parecida a la Figura 7.A.

Esta expresión permite evitar contar con la información correspondiente a los ciclos térmicos para poder correlacionar el nivel de tensiones residuales con un parámetro de proceso. Nuevamente, de los coeficientes obtenidos de este último ajuste se obtiene una expresión para correlacionar la tensión residual longitudinal máxima con la velocidad de avance, que se muestra en la Ecuación 4 y que tiene una curva prácticamente igual a la que se observa en la Figura 7.B.

$$
\sigma(\mathrm{U})=\left(-3,227 \frac{\mathrm{mm}}{\mathrm{rev}} \cdot \frac{514^{\mathrm{rev}} / \mathrm{min}}{\mathrm{U}}+85,809\right) \mathrm{MPa}
$$

Estas últimas expresiones presentan una utilidad tecnológica de interés permitiendo predecir el nivel de tensiones residuales en función de los parámetros de proceso empleados.

\subsection{Macrografías}

En la Figura 9 se observa una de las macrografías realizadas, correspondiente a la probeta 73 . En la misma se pueden apreciar las distintas zonas características de la soldadura por fricción agitación, la ausencia de defectos y una buena penetración, sin defectos de raíz. 


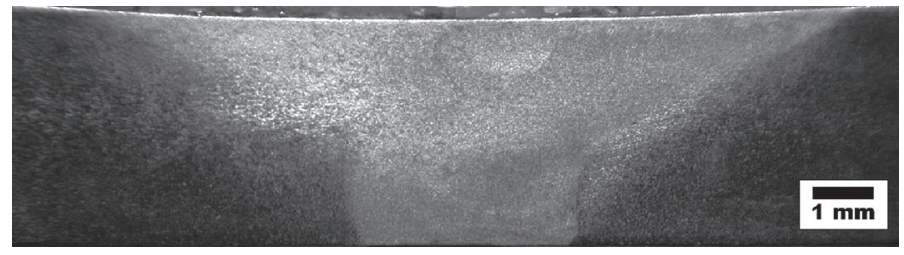

Figura 9. Macrografía de una junta soldada por FSW con una velocidad de avance de $73 \mathrm{~mm} . \mathrm{min}^{-1}$

\section{Conclusiones}

Se evaluaron las tensiones residuales obtenidas en uniones soldadas por FSW con distintas velocidades de avance, en chapa de $4 \mathrm{~mm}$ de espesor de AA7075-T651. Los valores de tensiones residuales longitudinales se relacionaron con aspectos del ciclo térmico generado durante la soldadura.

Las tensiones residuales máximas aumentaron con la velocidad de avance y fueron menores que las obtenidas en materiales similares. Las tensiones máximas se ubicaron en el centro del cordón y se encontraron entre 52 y $78 \mathrm{MPa}$, correspondientes a 10 a $15 \%$ de límite de fluencia del material.

A partir de los ciclos térmicos adquiridos se calcularon valores como la potencia neta y el calor aportado neto. Las temperaturas máximas medidas en el ciclo térmico disminuyen con la velocidad de avance, así como el calor aportado neto, mientras que la potencia neta se mantiene constante.

Al utilizar una herramienta con dimensiones menores a las comúnmente utilizadas para la configuración de junta analizada, resulta una potencia menor y se obtienen menores temperaturas máximas y tensiones residuales, siendo las dimensiones de la herramienta un factor relevante en la generación de tensiones residuales.

Se obtuvieron expresiones experimentales que relacionan la tensión residual longitudinal máxima con aspectos del ciclo térmico, como el calor aportado neto y temperatura máxima, y con parámetros del proceso como la velocidad de avance y el pitch. Si bien la tensión residual longitudinal máxima aumenta con la velocidad de avance, a partir de $100 \mathrm{~mm} \cdot \mathrm{min}^{-1}$ el aumento de la misma es marginal.

\section{Agradecimientos}

Los autores desean agradecer a INTI-Mecánica y a la Facultad de Ingeniería de la Universidad de Buenos Aires por el financiamiento y la utilización de sus instalaciones para el desarrollo del presente trabajo. Así también al personal de INTIMecánica y del Laboratorio de Materiales y Estructuras de FIUBA por la asistencia brindada.

\section{Referencias Bibliográficas}

[1] MISHRA, R. S., MA, Z. Y. Friction stir welding and processing. 2005, Materials Science and Engineering R, Vol. 50, p. 1-78

[2] LOMBARD, H., et al. Effect of process parameters on the residual stresses in AA5083-H321 friction stir welds. 2009, Materials Science and Engineering A, Vol. 51, p. 119-124

[3] LINTON, V. M., RIPLEY, M. I. Influence of time on residual stresses in friction stir welds in agehardenable $7 \mathrm{XXX}$ aluminium alloys. 2008, Acta Materialia, Vol. 56, p. 4319-4327.

[4] XU, W., LIU, J., ZHU, H. Analysis of residual stresses in thick aluminum friction stir welded butt joints. 2011. Materials and Design, Vol. 32, p. 2000-2005.

[5] FRATINI, L., ZUCCARELLO, B. An analysis of throughthickness residual stresses in aluminium FSW butt joints. 2006. International Journal of Machine Tools \& Manufacture, Vol. 46, p. 611-619.

[6] FULLER, C. B., et al. Evolution of microstructure and mechanical properties in naturally aged 7050 and $7075 \mathrm{Al}$ friction stir welds. 2010, Materials Science and Engineering A, Vol. 527, p. 2233-2240.

[7] MISHRA, R. S., MAHONEY, M. W. Friction Stir Welding and Processing. Ohio: ASM Internacional, 2007.

[8]AMERICAN SOCIETY FOR TESTING AND MATERIALS. ASTM E 8M: Standard Test Method for Tension Testing of Metallic Materials [metric], Pennsylvania, 2004. 24 p.

[9] ASM INTERNACIONAL. ASM Handbook: Volume 2, Properties and Selection: Nonferrous Alloys and SpecialPurpose Materials. 2.ed. Ohio, 1992.

[10] MOREIRA, P.M.G.P., et al. Residual stress evaluation of a MIG butt welded aluminium alloy plate, APAET Mecânica Experimental, Portugal, v.17, p.29-39, Jan. 2009.

[11] MASUBUCHI, K. Analysis of Welded Structures: Residual Stresses, Distortion, and their Consequences. 1.ed. New York: Pergamon Press, 1980. 642 p.

[12] MICRO MEASUREMENTS, B129-8: “Surface preparation for strain gage bonding", Vishay, 2010.

[13] ASM INTERNACIONAL. ASM Handbook: Volume 8, Mechanical Testing and Evaluation, 1.ed. Ohio, 2000.

[14] RADAJ, D. Heat Effects of Welding: Temperature Field, Residual Stresses, Distortion. 1.ed. Berlin: Springer-Verlag, 1992. 348 p.

[15] AMERICAN WELDING SOCIETY (AWS). Welding Handbook: Volume 1, Welding Science and Technology. 9.ed. Miami, 2001. 918 p. 